LIAMES 1 - pp. 61-73, Primavera 2001

\author{
Fernando Zúñiga \\ (Universidad de Zurich)
}

\title{
Dos Progresivos y dos Resultativos en el Mapudungun
}

\section{RESUMEN:}

El presente artículo ha esbozado cómo el mapudungún ha gramaticalizado dos verbos, meke'estar ocupado/a en una actividad' y müle- 'estar', en funciones que configuran un sistema de oposiciones complejas y que se relacionan estrechamente con el aspecto léxico de los predicados a los cuales se aplican. Los siete tipos de situaciones postulados para el mapudungún interactúan de tal manera con el aspecto gramatical que el sufijo -meke requiere poder referirse a fases dinámicas y es, por lo tanto, un progresivo similar al que se encuentra en muchas otras lenguas, mientras que -(kü)le funciona como progresivo con algunos tipos de situación (aquellos que sólo provean una fase dinámica en su representación semántica) y como resultativo con otros (aquellos que provean una fase estática y un límite inherente). Una forma que combina los sufijos -(kü)le y -we 'ya' parece referirse de modo específico tanto al estado o situación resultante como al evento que lo causó, estableciendo un nexo causal adicional a la semántica del predicado, y ha sido llamada aquí resultativo causal. De este modo, la lengua indígena hablada por el mayor número de personas actualmente en Chile viene a sumarse a otros idiomas de Asia y América que también presentan ya sea verbos cuyas formas son casos de polisemia o formas que con algunos predicados son progresivas y resultativas con otros.

\section{INTRODUCCIÓN}

El estudio del aspecto verbal de las lenguas indígenas sudamericanas ciertamente no ha sido tan productivo, al menos en términos de publicaciones, como la investigación de dicho campo en lenguas cuya descripción es comparativamente menos reciente y más extensa. En particular, recién están comenzando a aparecer análisis aspectuales de las lenguas del sur del continente de acuerdo a los modelos teóricos en boga desde la década pasada similares a los efectuados para las lenguas germánicas, románicas y eslavas. El presente artículo persigue el objetivo de continuar dando a conocer parte del sistema aspectual de la lengua indígena con mayor número de hablantes en Chile, el 
mapudungún ${ }^{1}$, en el espíritu de Golluscio (1998). Me he concentrado en la distinción hecha por la lengua entre dos diferentes maneras de referirse a una fase durante la cual la acción misma o su resultado son vigentes mediante las formas sintéticas con los sufijos -(kü)le y -meke, y a la distinción entre dos tipos de resultativos marcados formalmente por - $(k \ddot{u}) l e \mathrm{y}$ -(kü)le-we.

\section{BREVE ESBOZO DE LAMORFOSINTAXIS DELMAPUDUNGÚN}

El mapudungún es una lengua polsintética cuyo complejo verbal puede incluir un gran número de morfemas de carácter temporal, modal, aspectual, personal, locativo, etc., e incorporar sintagmas nominales y un reducido número de raíces verbales. Hay tres personas gramaticales (primera, segunda y tercera) y tres números (singular, dual y plural), pero la marca de número plural $p u$ normalmente no aparece en sintagmas nominales cuyos referentes no son seres humanos. Las relaciones gramaticales se marcan en el complejo verbal y no en los argumentos (es decir, corresponde al tipo head-marking según Nichols 1986, aun cuando la posesión nominal se marque a través de un pronombre posesivo junto al poseedor y no al elemento poseído). Hay dos tiempos, un futuro marcado con el sufijo - $a$ y un no futuro no marcado. La negación se marca en el verbo mediante los sufijos -la, - nu o - ki según el modo, y las formas verbales pueden ser finitas o no finitas / nominalizadas; en los ejemplos del presente artículo aparecen "infinitivos" en $-n$ y "participios" en -lu.

Considérese el siguiente ejemplo:

(1) Feymew rupan antü aku-rume-rke-y ruka mew

entonces pasado día llegar-de.pronto-CUOT-IND casa PPOS

tachi ülcha ñam-küle-lu, welu rume-fütra-ke-longko-nge-tu-rke-y.

ART joven.soltera perder-RES-PCP pero muy-largo-ns-cabello-ser-TU-CUOT-IND

'Entonces, pasado el día, llegó de repente, cuentan, a la casa de la muchacha soltera que estaba desaparecida, pero se había transformado, cuentan, en [persona de] cabellos muy largos.' (Salas 1992:218)

La preposición rupan 'después de' es el resultado de la lexicalización de la forma verbal no finita rupa-n 'pasado, pasando, pasar' (de rupa- 'pasar'). La postposición mew es de semántica subespecificada y puede significar, según el verbo utilizado y el contexto, 'en', 'a', 'por', 'desde', etc; la palabra feymew proviene de fey 'aquel, aquello' y mew 'después'. Las formas como la participial ñam-küle-lu 'que estaba desaparecida' serán objeto de repetida atención en lo que sigue del artículo. Finalmente, obsérvese cómo longko 'cabeza, cabello' se verbaliza mediante la sufijación de nge- 'ser', cómo se refiere al comienzo de dicho estado mediante el sufijo - $t u$ (de $t u$ - 'tomar, coger') y se modifica

'El mapudungún (mapu 'tierra' y dungu(n) 'habla, idioma'), también conocido como lengua mapuche (el nombre de la etnia, de mapu y che 'gente') o araucana, presenta una variación dialectal relativamente baja y se habla actualmente en el centro y sur de Argentina (unos 40.000 hablantes) y en la Región de la Araucanía en Chile (al menos unos 200.000 hablantes). Para más detalles ver Fabre (1998:720). 
mediante rume 'muy' y fütra 'grande, largo'; el sufijo -ke se utiliza en adjetivos atributivos cuando el número es no singular, es decir, dual o plural.

\section{LA CATEGORÍA GRAMATICAL ASPECTO EN MAPUDUNGÚN}

Introducción. Contrariamente a lo ocurrido en estudios sobre otras lenguas, donde las dimensiones temporal y aspectual tradicionalmente han sido analizadas conjunta y algo confusamente, las gramáticas de Augusta (1903) y Moesbach (1962) ya distinguieron el uso de morfemas como -(kü)le y -ke de aquel del sufijo de futuro - $a$. También Harmelink (1988), Smeets (1989) y Salas (1992) trataron por separado las categorías de aspecto y de tiempo, pero Golluscio (1998) fue pionera en el sentido de aplicar teorías aspectuales recientes al mapudungún.

Dichas teorías, articuladas claramente a partir de comienzos de la década pasada (Smith 1991, 1997; Sasse 1991a, 1991b; Breu 1994, 1996, 1997, 2000) se basan en la distinción entre ASPECTO LÉXICO y ASPECTO GRAMATICAL. El primero corresponde a las diferencias observadas por Vendler (1967) entre los diferentes predicados en inglés, p.ej. know 'saber', write 'escribir' y write the letter 'escribir la carta'. Su diferente comportamiento morfosintáctico pone en evidencia diferencias o bien presentes en su representación semántica pero invisibles en la superficie (entre know y write), o bien introducidas por otros elementos, p.ej. un sintagma nominal (entre write y write the letter). Mientras la forma *I am knowing '*estoy sabiendo' es agramátical en ambas lenguas, las formas I am writing 'estoy escribiendo' y I am writing the letter 'estoy escribiendo la carta' son correctas tanto en inglés como en castellano. Por otro lado, resulta claro que una aseveración como I was writing 'estaba escribiendo' necesariamente implica otra del tipo I wrote 'escribí', mientras que una aseveración como I was writing the letter 'estaba escribiendo la carta' no implica I wrote the letter 'escribí la carta' en ninguno de los dos idiomas. El aspecto se concibe, entonces, como la interacción entre el aspecto léxico, también llamado tipos de situación o aktionsarten (que distingue entre predicados como saber, escribir y escribir la carta, TS de aquí en adelante), y el aspecto gramatical o aspecto en el sentido estricto (que diferencia formas como escribía y escribí). Es frecuente utilizar el término ASPECTUALIDAD (del inglés aspectuality) para referirse a ambos componentes y el sistema que configuran.

La literatura que ha investigado diferentes aplicaciones de modelos basados en estos dos componentes teóricos, especialmente a lenguas indoeuropeas, es numerosa y no es del caso citarla aquí - el lector interesado puede consultar Dahl (ed.) (2000) tanto para estudios como para bibliografías. Difícilmente resulta sorprendente que el análisis de la aspectualidad en lenguas no indoeuropeas no haya avanzado a la par con el de los idiomas más accesibles, aun cuando Smith (1991, 1997), Sasse (ed.) (1991) y Breu (ed.) (2000) incluyan estudios sobre lenguas africanas, americanas y asiáticas. Una de las compilaciones más recientes en esta área es Ebert y Zúñiga (eds.) (en imprenta).

Aspecto léxico. Las preguntas relevantes en este ámbito son al menos dos: (a) Cuáles son los diferentes TS que deben postularse en la gramática universal y/o en una lengua dada, y (b) qué procedimientos permiten distinguirlos. Respecto de la primera, los lingüistas han procedido al análisis con la distinción de cuatro tipos de predicados de Vendler (1967) 
y han añadido uno, dos o tres más según la lengua particular o consideraciones teóricas más o menos a priori los llevasen a ello. El Cuadro 1 a continuación presenta las distinciones más frecuentemente halladas en estudios recientes:

\begin{tabular}{|c|c|c|c|}
\hline \multicolumn{4}{|c|}{ CUADRO 1: ASPECTO LÉXICO SEGÚN DIFERENTES AUTORES } \\
\hline \multicolumn{4}{|c|}{ tipos de situación originales según Vendler (1967) } \\
\hline Semántica & Sasse 1991a, 1991b & Breu 2000 & Smith 1991,1997 \\
\hline & totalmente estático & totalmente estático & estado \\
\hline$(\mathrm{L}) \sim \sim \sim \sim \sim(\mathrm{L})$ & actividad & actividad & actividad \\
\hline$\sim \sim \sim \sim \sim \sim \mathrm{L}$ & gradualmente & gradualmente & cumplimiento \\
\hline & terminativo & terminativo & \\
\hline $\mathrm{L}$ & totalmente & totalmente & logro \\
\hline & terminativo & terminativo & \\
\hline \multicolumn{4}{|c|}{ tipos de situación adicionales } \\
\hline$(\mathrm{L})-(\mathrm{L})$ & (estado) & relativamente estático & (estado) \\
\hline $\mathrm{L}$ & incoativo-estático & inceptivo-estático & - \\
\hline$\sim \sim \sim \sim \mathrm{L}$ & - & incoativo & - \\
\hline $\mathrm{L}(\longrightarrow)$ & $\begin{array}{c}\text { (actividad/totalmente } \\
\text { terminativo) }\end{array}$ & $\begin{array}{c}\text { (actividad/totalmente } \\
\text { terminativo) }\end{array}$ & semelfactivo \\
\hline
\end{tabular}

Una posible semántica de los diferentes TS ha sido representada gráficamente en la primera columna del Cuadro 1 mediante los símbolos para fase dinámica, —— para fase estática y $\mathrm{L}$ para límite, que puede ser inicial, final, o ambos. Los elementos entre paréntesis pueden estar presentes y es importante que puedan estarlo, pero son más bien elementos lógicos que semánticos. Así, los estados de Vendler corresponden a ——, las actividades a $\sim \sim$ , etc (la terminología de Smith sigue, a excepción del TS SEMELFACTivo, a Vendler)².

El presente artículo no puede proveer una discusión detallada del estado actual de la teoría aspectológica, como tampoco justificar exhaustivamente el uso que hace de Breu (2000) para el mapudungún. Baste remitir aquí a Zúñiga (en imprenta $)_{1}$ ) para una discusión acerca de por qué es preferible una taxonomía como la de Breu a la de Smith, así como más detalles acerca de los tests específicos que permiten distinguir los TS siguientes:

(2) Aspecto léxico en el mapudungún: tipos de situación
a. totalmente estático
(L)- (L)
p.ej. wentru-nge
'ser (un) hombre'
b. relativamente estático
(L) (L)
p.ej. müle-
'estar'
c. actividad
p.ej.lef-
d. cumplimiento
$\sim \sim \sim \sim \mathrm{L}$
p.ej. ruka-
'correr'
e. inceptivo-estático
$\mathrm{L}$
p.ej. kurü-
f. logro
$\mathrm{L}$
p.ej. chafo-
'construir una casa'
g. difásico

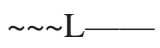
p.ej. ñam-
'ennegrecerse'
'toser'
'perderse'

${ }^{2}$ Es importante observar que los diferentes autores definen los TS sobre la base de distintos elementos o parámetros, como p.ej. [ \pm télico], $[ \pm$ dinámico] y [ \pm durativo] (Smith, siguiendo a Vendler), y que las correspondencias entre las categorías según los diferentes autores responden más bien a necesidades expositivas que a una comparación teórica rigurosa. 
En cuanto al comportamiento morfosintáctico, baste aquí observar lo siguiente: 1) son combinables con -(kü)le: $\mathrm{c}, \mathrm{d}$, e y g; 2) son combinables con -meke: $\mathrm{c}, \mathrm{d}$ y g, y 3) las formas en -(kü)le y en -meke tienen diferente significado para el TS g.

Si bien muchos predicados no presentan marca morfológica alguna que permita decidir a qué clase pertenecen (p.ej. kim- 'saber', lef- 'correr', etc.), algunos morfemas están claramente relacionados con TS específicos:

a) -nge, posiblemente relacionado con el verbo $n g e$ - 'ser' deriva predicados estáticos de raíces o sintagmas nominales o adjetivales: karü 'verde' - karü-nge-y 'es verde'; wentru 'hombre' - wentru-nge-y 'es (un) hombre'.

b) - $t u$, relacionado con el verbo $t u$ - 'tomar, coger', tiene una multitud de funciones que, sin embargo, no son impredecibles. Con verbos de movimiento como $a k u$ - 'llegar', significa 'de vuelta', p. ej. aku-tu-y 'llegó de vuelta' (considérese también amu-tu-y 'se fue' de amu- 'ir'). Con los verbos denominales derivados según a), significa el comienzo del estado de manera puntual: wentru-nge-tu-y 'se convirtió en (un) hombre'; los adjetivos no precisan esta derivación y significan el comienzo del estado sin marca alguna: karü$y$ 'se volvió verde', como se ve más adelante. Algunas raíces verbales ocurren sólo con el sufijo - $t u$ sin que haya oposición con una forma no marcada, p.ej. papel-tu- 'estudiar'. Algunos verbos aparecen de modo alternativo con otras pocas raíces verbales que telifican, p.ej. umaw-tu- umaw-nag- 'quedarse dormido/a' (nag- 'bajar, descender').

c) La iteración de la raíz y la sufijación de -nge o - tu tiene como consecuencia una forma de significado iterativo (una actividad), p.ej. rüngkü- 'saltar' - rüngkü-rüngkü-tu-y 'dio saltos'.

Aspecto gramatical. Al menos desde Comrie (1976) es usual distinguir dos puntos de vista desde los cuales se puede describir una situación dada: IMPERFECTIVO y PERFECTIVO. El primero dice relación con una descripción "desde dentro", tomando en cuenta la "constituencia interna" de la situación, mientras el segundo contempla la situación "desde fuera". La perspectiva imperfectiva puede además subdividirse en diferentes tipos: habitual, progresivo, etc. También Smith $(1991,1997)$ opera con dicha distinción, la cual considera parte de la gramática universal. En el contexto de modelos de aspectualidad que operan con el concepto de SELECCIÓN, las formas imperfectivas seleccionan fases y las perfectivas límites.

Si el objetivo final es llevar a cabo un análisis émico de las categorías del mapudungún, resulta importante notar los siguientes aspectos de la morfología verbal: (a) existe una forma no marcada; (b) existen formas marcadas con - $(k \ddot{u}) l e$ y -meke, y (c) existen formas marcadas con -ke, que puede aparecer con o sin los sufijos mencionados (b); este último es de origen desconocido, significa que la acción se realiza de un modo habitual, y no será objeto de estudio detallado aquí.

El siginificado de las formas en -(kü)le y -meke depende del aspecto léxico según se aprecia en el Cuadro $2^{3}$ :

\footnotetext{
${ }^{3}$ También existe un progresivo analítico, al parecer más reciente, formado con petu 'todavía' y el verbo no marcado, p.ej. petu lef- $i$ 'está corriendo', de significado indistinguible de los otros dos y correspondiente, a grandes rasgos, a la forma en -meke en cuanto a su distribución. Esta forma no se analiza en este artículo.
} 
CUADRO 2: INTERACCIÓN DE ASPECTOS GRAMATICAL Y LÉXICO

\begin{tabular}{c|c|c|c}
\hline Tipo de situación & $-\varnothing$ & $-(k \ddot{u}) l e$ & - meke \\
\cline { 2 - 3 }$\left(\mathrm{L}_{1}\right)-\left(\mathrm{L}_{2}\right)$ & - & - & - \\
$\left(\mathrm{L}_{1}\right) \sim \sim \sim \sim$ & - & - \\
$\sim \sim \sim \sim \mathrm{L}$ & $\mathrm{L}$ & $\sim \sim \sim$ & $\sim \sim \sim$ \\
$\mathrm{L}-\sim \sim \sim \sim$ & - \\
$\mathrm{L}$ & $\mathrm{L}$ & $\mathrm{L}$ & - \\
$\sim \sim \sim \sim \sim \sim$ \\
\hline
\end{tabular}

Las formas en - $(k \ddot{u}) l e \mathrm{y}$-meke corresponden a primera vista a operadores aspectuales imperfectivos, por focalizar, seleccionar o enfatizar, según el modelo teórico concreto que se prefiera, fases (—— o ) y no límites (L). Si bien con algunos TS la forma no marcada correspondería a una perspectiva perfectiva (aquellos donde hay fases dinámicas o límites inherentes), con otros correspondería a una perspectiva imperfectiva (donde hay sólo fases estáticas y/o límites posibles pero "externos", no inherentes), por lo cual lo más apropiado parece ser hablar de una forma NEUTRAL ${ }^{4}$. Los sufijos - $(k \ddot{u}) l e$ y -meke serán objeto del próximo capítulo.

Es importante resaltar la diferencia fundamental entre la caracterización de algunos lexemas en mapudungún y aquella de lexemas "equivalentes" en lenguas románicas.

A. Los adjetivos como kurï 'negro' pueden verbalizarse sin necesidad de morfología adicional (lo cual es característico de los adjetivos en general), y entonces siempre significan 'comenzar a tener la cualidad X', como es el caso de kurü- 'ennegrecerse'. Una vez que rige el estado, se hace referencia a él con una forma en -(kü)le, p.ej. kurü-le-y 'está negro'. Si en vez de un estado potencialmente transitorio se trata de una característica permanente, se sufija -nge, p.ej. kurü-nge-y 'es negro', en cuyo caso se ha modificado el TS de inceptivo-estático L_—a totalmente estático

B. Cumplimientos como af- 'terminar' tienen formas en -(kü)le y -meke sinónimas, es decir, af-küle-y $=a f-m e k e-y$ 'está terminando'. La forma no marcada se refiere a la trasgresión del límite inherente, es decir, $a f-i$ 'terminó'. Diferentes son los verbos difásicos como el ya citado ñam-, cuyas diferentes formas presentan los siguientes significados: ñam-meke-y 'está perdiéndose, desapareciendo', ñam-i 'se perdió', ñam-küle-y 'está perdido/a'. En otras palabras, la representación de predicados como af- difiere de la de predicados como $\tilde{n} a m$ - en el sentido de que la fase posterior LóGICAMENTE presente en el caso de $a f$ - está también en la SEMÁNTICA de ñam-.

\section{PROGRESIVIDADY RESULTATIVIDADENMAPUDUNGÚN}

Al igual que con otros sufijos gramaticalizados a partir de verbos independientes como el andativo -miaw (de miaw- andar), -künu y -nie (de künu- 'dejar' y nie- 'tener'), los

\footnotetext{
${ }^{4}$ Varias teorías, p.ej. la de Smith $(1991,1997)$, consignan el aspecto neutral como parte integrante de los sistemas aspectuales.
} 
dos morfemas objeto del presente capítulo conservan una buena parte de su significado original. El lector interesado en más detalles deberá consultar Zúñiga (en imprenta, en imprenta $_{2}$ ). Antes de discutir ambos sufijos por separado, obsérvense los ejemplos a continuación; (3a) muestra un ejemplo de -meke con una actividad iterativa (lüpef-lüpeftu- 'abrir y cerrar los ojos una y otra vez'), (3b) uno de -(kü)le con dos actividades (ülkantu- 'cantar' y wiraf- 'galopar') y un cumplimiento (küpa- 'venir'), y (3c) uno con un predicado inceptivo-estático (ngolli- 'embriagarse'):

(3) a. Pepi-nengüm-la-y ngürü. Lüpef-lüpef-tu-meke-y müten. poder-moverse-NEG-IND zorro cerrar.y.abrir.los.ojos-ITER-TU-PROG-IND solamente 'El zorro no pudo moverse. Estuvo abriendo y cerrando los ojos una y otra vez nada más.' (Salas 1992:294)

b. Ülkantu-le-y ngürü, küpa-le-y wiraf-(kü)le-y. cantar-PROG-IND zorro venir-PROG-IND galopar-PROG-IND 'El zorro estaba cantando, viniendo, galopando.' (Salas 1992:303)

c. Feymew ngolli-ngün. Ngolli-le-lu engün lukatu-w-i-ngün. entonces embriagarse-3p embriagarse-RES-PCP 3p insultar-REFL-IND-3p 'Entonces se embriagaron. Estando ebrios, se insultaron unos a otros.'

(Salas 1992:263)

-meke. Golluscio (1998) propuso un análisis bimorfémico de este sufijo: andativo -me seguido del habitual $-k e$. Si este análisis fuese correcto, dadas las posiciones de dichos morfemas en el complejo verbal, debería haber formas con significado progresivo que coocurrieran con otros morfemas en posiciones intermedias (p.ej. -uye, -ka, -pe), lo cual parece no ser el caso. Además, formas que sufijasen simultáneamente -meke y -ke no deberían ser posibles, pero al menos algunos hablantes juzgan aceptables ejemplos como (4a), si bien parecen ser preferibles formas en -(kü)le-ke. Finalmente, obsérvese que existe el verbo meke- 'estar ocupado/a haciendo algo', y con frecuencia ambas construcciones tienen un significado prácticamente equivalente, como en $4 \mathrm{~b}$.
(4) a. Katrü-ka-meke-ke-y ilo. (también: katrü-ka-le-ke-y ...) cortar-KA-PROG-HAB-IND carne
'Siempre está (ocupado/a) cortando la carne en pedazos.'
b. Iñche meke-n tañi kutran-tu-n. 1s estar.ocupado/a-1s 1s POS enfermedad-TU-INF
Iñche kutran-tu-meke-n.
1s enfermedad-TU-PROG-1s
'Estoy sufriendo.' (Smeets 1989:376)

Por las razones expuestas anteriormente me he inclinado por un análisis monomorfémico del sufijo -meke. 
Su significado, como ya se mencionó en el capítulo precedente, normalmente corresponde a la construcción estar $V$-ndo en castellano o portugués brasileño y puede caracterizarse como PROGRESIVO. Es incompatible tanto con los verbos estrictamente puntuales (logros) como los estáticos, y con los predicados que admiten una fase dinámica previa y una fase estática posterior, -meke invariablemente se refiere a la fase dinámica. Pienso que no hay argumentos contundentes que contradigan la hipótesis de que tal especialización dice relación con el origen del morfema; el significado de 'estar ocupado/a realizando una acción determinada' es naturalmente incompatible tanto con situaciones como 'ser (un) hombre' como con aquellas que por su duración no admiten una extensión temporal. Tampoco es posible construir formas en -meke a partir de predicados inceptivo-estáticos.

-(kii)le. Este sufijo probablemente ha resultado de la gramaticalización del verbo müle'estar's. Dado que se desconoce el desarrollo diacrónico exacto seguido por este morfema, es aventurado suponer que su aplicación estuviera restringida en un comienzo a algunos tipos de predicados, p.ej. los estáticos, para después extenderse su uso a los dinámicos. Actualmente, todas las clases de predicados aceptan la combinación con -(kü)le excepto los puntuales y los estáticos como wentru-nge- 'ser (un) hombre' ${ }^{6}$ o nie- 'tener'.

Nuevamente, el significado de - $(k \ddot{u}) l e$ y su aplicación pueden explicarse sobre la base del origen "estático" del sufijo. Combinado con verbos que incluyan en su representación semántica sólo una fase dinámica, se refiere a ella, quizás como producto de una extensión de su aplicabilidad. Pero cuando el predicado presenta tanto una fase dinámica previa como una fase estática posterior, - $(k \ddot{u}) l e$ invariablemente se refiere a la segunda; de modo similar, si el predicado consiste solamente de un límite inherente y una fase estática posterior, -(kü)le se refiere a la fase. Así, este morfema tiene una función PROGRESIVA con unas clases de predicados y RESULTATIVA con otras. En forma menos rigurosa pero abreviada se le podría llamar PROGRESIVO ESTÁTICO para enfatizar el contraste con la dinamicidad inherente de $-m e k e^{7}$. No es posible construir formas en - $(k \ddot{u}) l e$ a partir de verbos estáticos o puntuales (logros).

\footnotetext{
${ }^{5}$ Obsérvese que el alomorfo -küle después de no-vocales es exactamente paralelo al sufijo ambulativo -miaw, más transparente aún (verbo miaw- 'andar', alomorfo después de no-vocales -kiaw).

Smeets (1989:372) registra algunos casos en que, análogamente al inglés, formas como witrannge-le-pa-y =s/he is being a visitor here 'está (actuando) como visitante aquí' son aceptadas por algunos hablantes. No obstante, formas como la anterior parecen ser extremadamente infrecuentes, pero aunque no lo fueran podrían analizarse como las formas continuas en inglés del tipo you're being very stupid now 'te estás comportando de manera muy estúpida en estos momentos'.

7 Es frecuente que se asocie "progresividad" a "dinamicidad", y en este sentido la nomenclatura impresionista propuesta aquí resultaría redundante en el caso de PROGRESIVO DINÁMICO y contradictoria en el caso de PROGResivo estático. Sin embargo, es discutible en qué medida se puede hablar de "progreso" de la acción en actividades como correr o dormir (atélicas, sin sintagma nominal que introduzca medida alguna).

Si la dinamicidad es condición necesaria para la progresividad en el sentido de que "debe ocurrir algo", es preferible caracterizar - $(k \ddot{u})$ le como un caso de polisemia: progresivo y resultativo.
} 
Obsérvense asimismo los ejemplos en (5). La raíz longko significa 'cabeza, cabellos, espiga' en su variante nominal (con su frecuente acepción de 'cacique, jefe'), y puede tener diversos significados al estar verbalizada. Particularmente notable es que no se pueda utilizar la forma no marcada (5b) para significar que alquien "comenzó a estar a la cabeza”, lo cual sí se puede decir con la forma longko-le-we-y 'quedó a la cabeza' (formalmente una forma en -(kü)le; se dirá más acerca de esta forma más adelante).
a. longko-le-y
b. longko-y
'está a la cabeza'
(*longko-nge-y)
c. longko-nag-küle-y
'espigó'
d. fütra-longko-tu-le-y
'está con la cabeza hacia abajo' (nag- 'descender')
e. longko-tu-y
'tiene los cabellos largos' (fütra 'grande, largo', Ejemplo 1) 'recibió un golpe en la cabeza'

Ambigüedades. Se ha observado que en algunas lenguas hay predicados que, al aparecer en la forma progresiva, pueden significar tanto una fase dinámica previa como una fase estática posterior (Ebert 1995, Shirai 1998, Breu 2000, Roos en imprenta), ya sea en forma generalizada como en japonés (6a) o sólo con algunos verbos como en mandarín (6b).

(6) a. Japonés

Ken-wa seetaa-o ki-te i-ru.

K.-TOP suéter-AC vestir-PROG PROG-NPT

'Ken se está poniendo / tiene puesto un suéter.' (Shirai 1992:672)

b. Mandarín
Tâ
chuân-zhe
dàyî.
$3 \mathrm{~s}$ vestir-PROG
abrigo
'Se está poniendo / tiene puesto un abrigo' (Roos en imprenta)

Es más, ni siquiera hay que ir tan lejos, ni geográfica ni tipológicamente, para encontrar casos similares de "ambigüedad". Mientras algunos pares aspectuales rusos como umirat'/umeret' 'morir' y lovit'/pojmat' 'coger' pueden ser considerados del tipo cumplimientos L otros como prjatat'sja/sprjatata'sja 'esconder(se)' y el verbo italiano indossare 'ponerse, tener puesto' son difásicos del tipo L_— porque el mismo operador aspectual puede referirse tanto a la fase previa como a la posterior -

Asimismo, en otros idiomas se emplea la misma forma para significar progresividad con algunas clases de verbos y resultatividad con otras, como en algunas lenguas turcas (ejemplificadas aquí por el karachay) y en forma bastante generalizada en las lenguas iroquesas (representadas aquí por el cayuga):

\section{(7) a. Karachay}

$\begin{array}{llll}\text { Aè-ïb } & \text { turadï. / } & \text { Tig-ib } & \text { turadi. } \\ \text { 'abrir-GER } & \text { es(tá) } & \text { coser-GER es(tá) } \\ \text { 'Ha cerrado.' } & & \text { 'Está cosiendo' (Nedjalkov \& Nedjalkov 1987) }\end{array}$


b. Cayuga

Ak'ní:khõ'

/ Tewakatská'hõ'.

1s:coser:PERF

DU:1s:masticar:PERF

'Lo he cosido.'

'Estoy masticando.'

Aun cuando el presente estudio no tiene por objeto localizar al mapudungún en una tipología universal de la ambigüedad en el terreno de la progresividad / resultatividad, resulta interesante observar de modo tentativo que algunas lenguas (tipo I, japonés) toleran una ambigüedad sistemática de formas que pueden significar lo uno o lo otro según el contexto, mientras otras (tipo II, mandarín, ruso, italiano) limitan las formas ambiguas a algunos pocos predicados, entre los cuales 'vestir(se)', 'esconder(se)' y otros pocos más parecen ocupar un lugar prototípico. Otras lenguas (tipo III, karachay, cayuga) no tienen predicados ambiguos sino formas que, según de qué verbo se trate, tienen función progresiva o resultativa. El mapudungún parece ser un caso especial de este tercer tipo de idiomas en el sentido de que las formas en -meke y aquellas en -(kü)le son sinónimas mientras el predicado no distinga en su semántica una fase previa de una fase posterior, en cuyo caso ambas formas se "especializan". Mayor rigor en este esbozo tipológico, así como un mayor número de lenguas que presenten fenómenos similares, serán características de investigación futura en este campo.

-(kii)le-we. Una forma particularmente interesante es la que combina el resultativo -(kü)le con el morfema -we. Como adjetivo, we significa 'nuevo, joven, fresco', y se puede incluir en el complejo verbal con el significado de 'ya', aproximadamente equivalente a la partícula $\operatorname{dew}(m a)$, p.ej. en (8a), y también conjuntamente con la negación con función cesativa, o sea, de significado 'ya no', p.ej. en (8b).

(8) a. Karü-we-y ta ilo. verde-wE-IND ART carne

'La carne ya está verde (=abombada).' (Golluscio 1998:43)

b. Küpa-we-la-ya-y.

venir-wE-NEG-FUT-IND

'Ya no vendrá.'

Como simulfijo, -(kü)le-we significa 'quedar en un estado determinado o realizando una acción determinada (como consecuencia de algo)', p.ej. kidu-le-we-y 'quedó solo' ( kidu 'solo') o kansa-le-we-y 'quedó cansado' (kansa- 'cansarse'). A modo de un ejemplo más, -duam, un morfema de significado algo vago que dice relación con actividad mental y/o emocional en general, puede combinarse con af- 'terminar' para formar af-duam'sorprenderse' (TS inceptivo-estático) y raki- 'contar' para formar raki-duam- 'pensar' (TS actividad); obsérvense al respecto los ejemplos en (9):

(9)

$\begin{array}{clllll}\text { a. } & \text { Fey kisu } & \text { ñi } & \text { ñuke } & \text { petu } & \text { püru-rke-y. } \\ 3 & \text { propio/a } & \text { 3POS } & \text { madre } & \text { PROG bailar-CUOT-IND } \\ \text { Afduam-küle-we-pu-y } & \text { pe-lu } & \text { tañi } & \text { chum-meke-n tañi ñuke. }\end{array}$

soprenderse-KÜLE-WE-TRANS-IND ver-PCP 3POS hacer.qué-PROG-INF 3POS madre 'Fue advertido que su propia madre en ese momento estaba bailando. Se quedó sorprendido allá viendo lo que estaba haciendo su madre.' (Salas 1992:272) 
Zúñiga: Dos Progresivos y dos Resultativos en el Mapudungún

$\begin{array}{llcc}\begin{array}{l}\text { b. Feymew } \\ \text { entonces }\end{array} & \begin{array}{l}\text { kom-küle-ka-rke-y. } \\ \text { todo-RES-CONT-CUOT-IND }\end{array} & \begin{array}{c}\text { Feymew } \\ \text { entonces }\end{array} & \begin{array}{c}\text { rakiduam-küle-we-y } \\ \text { pensar-KÜLE-wE-IND }\end{array} \\ \begin{array}{l}\text { chumngelu } \\ \text { porqué }\end{array} & \text { ñi } \quad \text { apo-le-ka-n } & \text { chi } & \begin{array}{l}\text { muday. } \\ \text { chicha }\end{array}\end{array}$

'Entonces resultó que seguía estando toda [la chicha]. Entonces se quedó pensandoporqué seguía lleno [el cántaro] de chicha.' (Salas 1992:262)

Salas (1992:188) describe la diferencia entre la forma en -(kü)le y aquella en (kü)le-we caracterizando a la primera como DURATIVA (además de progresiva) y sólo a la segunda como RESULTATIVA, ya que las formas en -(kü)le-we significan no solamente que algo o alquien se encuentra en un determinado estado, sino que dicho estado es consecuencia o resultado de algún evento. Dicha nomenclatura presenta algunos inconvenientes, sin embargo. En primer lugar, el término DURATIVo es frecuentemente empleado para referirse a aktionsarten o elementos de las mismas, y no a aspectos gramaticales. Además, el término Resultativo puede entenderse de modo general o de modo específico; de modo general significa que un estado determinado es el resultado de algún evento de manera directa o indirecta, y en este sentido dice relación más estrecha con la forma en -(kui)le-we; de modo específico, por el contrario, un estado determinado es el resultado de un evento sólo si "forma parte de la caracterización de un cierto tipo de evento [=tipo de situación, FZ] al cual pertenece dicho evento" (Dahl 1985:135, mi traducción). En otras palabras, la forma en -(kü)le resalta el estado que ha resultado de un evento hasta cierto punto trivial pero absolutamente necesario, o sea, el comienzo de dicho estado, mientras que la forma en -(kü)le-we se refiere al estado resultante sin dejar demasiado de lado el evento que lo causó, pero además implica que relacionado con dicho evento hay otro que no es trivial, presente en el contexto lingüístico o paralingüístico, y establece una relación causal entre ambos ausente de la semántica estricta de los predicados en cuestión. En (9) arriba, por ejemplo, se utilizan las formas en -(kü)le-we a continuación de la descripción de los eventos que han causado ya sea un estado (el estar sorprendido en 9a) o una actividad (el pensar en 9b), porque las formas simples afduam-küle-y 'está sorprendido' o rakiduam-küle-y 'está pensando' no explicitarían que las fases a las que se refieren los predicados fueran la consecuencia de algo en particular. Por lo anterior, he preferido llamar RESULTATIVO a la forma relativamente simple en -(kü)le y caracterizar la forma en -(kü)le-we de manera tentativa como RESULTATIVO CAUSAL.

\section{REFERÊNCIAS BIBLIOGRÁFICAS}

AUGUSTA, Félix José de. (1903) [1990]. Gramática mapuche bilingüe. Santiago: Séneca.

BREU, Walter. (1994). Interactions between lexical, temporal and aspectual meanings. Studies in Language 18.1: 23-44.

.(1996). Komponentenmodell der Interaktion von Lexik und Aspekt. En W. Girke (ed.): Slavistische Linguistik 1995. Referate des XXI Konstanzer Slavistischen Arbeitstreffens, pp. $37-$ 74. Munich: Otto Sagner. [=Slavistische Beiträge 342]. 
.(1997). Komplexe aktionale Verbklassen, insbesondere Inchoativa. En T. Berger \& J. Raecke (eds.): Slavistische Linguistik 1997. Referate des XXIII Konstanzer Slavistischen Arbeitstreffens, pp. 55-80. Munich: Otto Sagner. [=Slavistische Beiträge 375].

.(2000). Zur Position des Slavischen in einer Typologie des Verbalaspekts (Form, Funktion, Ebenenhierarchie und lexikalische Interaktion). En Breu (ed.), pp. 21-54.

, Walter (ed.). (2000). Probleme der Interaktion von Lexik und Aspekt (ILA). Tübingen: Niemeyer.

COMRIE, Bernard. (1976). Aspect. Cambridge: Cambridge University Press.

DAHL, Östen. (1985). Tense and aspect systems. Oxford: Blackwell.

.(ed.). 2000. Tense and aspect in the languages of Europe. Berlín / Nueva York: Mouton de Gruyter.

EBERT, Karen H. (1995). Ambiguous perfect-progressive forms across languages. En Pier Marco Bertinetto, Valentina Bianchi, Östen Dahl \& Mario Squartini (eds.): Temporal reference. Aspect and actionality. Vol. 2: Typological perspectives, pp. 185-203. Turín: Rosenberg \& Sellier.

EBERT, Karen H. \& ZÚÑIGA, Fernando (eds.). en imprenta. Aktionsart and aspectotemporality in non-European languages. Zurich: ASAS-Verlag, Universität Zürich.

GOLLUSCIO, Lucía. (1998). Aspecto verbal en mapudungun. En Lucía Golluscio \& Yosuke Kuramochi, eds.: Lingüística y literatura mapuche. Aproximaciones desde ambos lados de los Andes. Trabajos del I Simposio Binacional de Lingüística y Literatura Indígenas. Temuco, 23-25 de octubre de 1995. Temuco / Buenos Aires: Universidad Católica de Temuco, Universidad de Buenos Aires.

HARMELINK, Bryan. (1988). The expression of temporal distinctions in Mapudungun. Lenguas Modernas 15:125-130.

NEDJALKOV, Igor \& NEDJALKOV, Vladimir. (1987). Karačaevo-balkarskaja glagol'naja forma na $b /-p$ tur- $a$ - so značenijami nastojaščego i prošedšego vremeni (v sravnenii $\mathrm{s}$ formami na $-b$ tura / tur-ib- v uzbekskom jazyke. Funkcional'no-semantičeskie aspekty grammatiki, pp. 113-121. Moscú : Nauka.

NICHOLS, Johanna. (1986). Head-marking and dependent-marking grammar. Language $62.1: 65-119$

ROOS, Olivier. (en imprenta). -zhe in Mandarin Chinese. En Ebert \& Zúñiga (eds.).

SALAS, Adalberto. (1992). El mapuche o araucano. Fonología, gramática y antología de cuentos. Madrid : Editorial MAPFRE.

SASSE, Hans-Jürgen. $\left(1991^{\mathrm{a}}\right)$. Aspekttheorie. En Sasse (ed.), pp. .(1991b). Aspect and aktionsart: a reconciliation. En Carl Vetters y Willy Wandeweghe (eds.): Perspectives on aspect and aktionsart, pp. 31-46. Bruselas: Editions de 1'Université de Bruxelles. [=Belgian Journal of Linguistics 6].

SASSE, Hans-Jürgen (ed.). (1991). Aspektsysteme. Colonia: Arbeitspapier No. 14 (Neue Folge) des Instituts für Sprachwissenschaft, Universität zu Köln.

SHIRAI, Yasuhiro. (1998). Where the progressive and the resultative meet. Imperfective aspect in Japanese, Chinese, Korean and English. Studies in Language 22.3: 661-692.

SMEETS, Ineke. (1989). A Mapuche grammar. Ph. D. dissertation, Rijksuniversiteit te Leiden.

SMITH, Carlota. (1991). The parameter of aspect. Dordrecht: Kluwer. .(1997). The parameter of aspect. 2a ed. Dordrecht: Kluwer. 
VENDLER, Zeno. (1967). Verbs and times. En Linguistics in philosophy, pp. 97-121. Ithaca / Londres: Cornell University Press

ZÚÑIGA, Fernando. en imprenta ${ }_{1}$. A selection theory of Mapudungun aspect. En Ebert \& Zúñiga (eds.). .en imprenta ${ }_{2}$. Mapudungun. Languages of the Wolrd/Materials 376. Munich: Lincom Europa.

\section{ABREVIATURAS}

$\begin{array}{ll}\text { AC } & \text { acusativo } \\ \text { ART } & \text { artículo } \\ \text { CONT } & \text { continuativo } \\ \text { CUOT } & \text { cuotativo } \\ \text { DU } & \text { duálico } \\ \text { GER } & \text { gerundio } \\ \text { HAB } & \text { habitual } \\ \text { IND } & \text { indicativo } \\ \text { INF } & \text { infinitivo } \\ \text { NEG } & \text { negación } \\ \text { NPT } & \text { no-pretérito } \\ \text { ns } & \text { no-singular } \\ \text { p } & \text { plural } \\ \text { PART } & \text { partícula } \\ \text { PCP } & \text { participio } \\ \text { PERF } & \text { perfecto } \\ \text { POS } & \text { posesivo } \\ \text { PPOS } & \text { postposición } \\ \text { PROG } & \text { progresivo } \\ \text { REFL } & \text { reflejo } \\ \text { RES } & \text { resultativo } \\ \text { S } & \text { singular } \\ \text { TOP } & \text { tópico } \\ \text { TRANS } & \text { translocativo } \\ & \end{array}$

